\title{
APPORTS RELATIFS EN NOURRITURES NATURELLE ET ARTIFICIELLE DANS L'ALIMENTATION DU TILAPIA OREOCHROMIS NILOTICUS EN CAPTIVITE
}

\author{
Y. BAMBA (1), A. OUATTARA (1), J. MOREAU (2) ${ }^{\star}$ G. GOURENE (1)
}

(1) Laboratoire d'Environnement et de Biologie Aquatique (LEBA), UFR des Sciences et Gestion de l'Environnement, Université d'Abobo-Adjamé, 02 Bp 801 Abidjan 02 (Côte d'Ivoire).

(2) Laboratoire d'Ecologie Fonctionnelle, UMR 5245 CNRS/UPS/INPT ENSAT. Avenue de l'Agrobiopôle. BP 107 Auzeville Toulouse 31326 Castanet Tolosan France.

* Auteur correspondant : courriel : jmoreau@ensat.fr

Reçu le 12 juillet 2007

Accepté le 16 octobre 2007

Received July 12, 2007

Accepted October 16, 2007

\section{RESUME}

Des études sur le rythme alimentaire, la ration alimentaire journalière et la quantité des nourritures naturelles et artificielles consommées ont été réalisées chez des populations d'alevins $(6 \mathrm{~g})$, de juvéniles (30 g) et d'adultes $(250 \mathrm{~g})$ d'Oreochromis niloticus. L'échantillonnage a été effectué toutes les 3 heures, sur deux cycles de $24 \mathrm{~h}$ dans 4 bassins bétonnés (alevins) et dans 8 étangs (juvéniles et adultes). Le contenu des estomacs a été analysé. Les poids frais du contenu des estomacs ont été traités par le logiciel MAXIMS pour déterminer les taux d'ingestion, d'évacuation et la ration alimentaire journalière.

Les courbes de la prise de nourritures présentent deux pics montrant une activité alimentaire périodique et diurne. En dehors des périodes de nourrissage (9h et 15h), le contenu des estomacs est composé majoritairement d'aliments naturels chez les alevins et juvéniles. En revanche, chez les adultes, les nourritures apportées sont plus abondantes entre $9 \mathrm{~h}$ et $24 \mathrm{~h}$. En dehors de cette période, le bol alimentaire est constitué presque uniquement de nourritures naturelles chez toutes les populations des poissons prélevés. Les rations alimentaires distribuées quotidiennement estimées sont de $10 \% ; 6,7 \%$ et $3,7 \%$ du poids corporel respectivement chez les alevins, les juvéniles et les adultes. Dans ce même ordre des populations indiquées, les nourritures naturelles consommées ont été approximativement de $4 \%, 2,31 \%$ et $1 \%$ contre $6,4,45$ et 2,7 \% pour l'aliment artificiel.

Mots-clés : Oreochromis niloticus, pisciculture semi-intensive, contenu stomacal, nourritures naturelles, alimentation artificielle, Côte d'Ivoire.

\section{RELATIVE CONTRIBUTIONS OF NATURAL AND SUPPLEMENTAL FOOD IN NILE TILAPIA OREOCHROMIS NILOTICUS FEEDING IN CAPTIVITY}

\section{ABSTRACT}

Studies on feeding periods, daily ration and relative artificial and natural food consumption in populations of juveniles $(6 \mathrm{~g})$, sub-adult $(30 \mathrm{~g})$ and adults $(250 \mathrm{~g})$ of Oreochromis niloticus were realized. Sampling took place over two 24-hour cycles in four 
concrete basins (juvenile) and eight ponds (sub-adult and adult). Fish were caught at three hourly intervals. Stomach contents of different populations were microscopically analysed. The gut content weights were submitted to the feeding model MAXIMS to determine the ingestion, evacuation rates and daily ration. Two peak feeding periods were evident. The fish showed diurnal diel feeding periodicity. Outside the food distribution periods $(9 \mathrm{~h}$ and $15 \mathrm{~h}$ ), the gut contents were mostly composed of natural food in juvenile and sub-adult populations. In addition, in adult population, artificial food was more important from $9 \mathrm{~h}$ to $24 \mathrm{~h}$. outside this period, the gut content in each fish population was only constituted of natural food. The daily rations were about $10 \%, 6.7 \%$ and $3.7 \%$ respectively for the juvenile, sub-adult and the adult. In that order, the consumed natural food represented $4 \% 2.3 \%$ and $1 \%$. Respective values of the artificial food estimated were $6 \%, 4.45 \%$ and $2.7 \%$ for the juvenile, sub-adult and the adults.

Key-words : Oreochromis niloticus, semi-intensive aquaculture, gut content, natural food, supplemental feeding, Côte d'Ivoire.

\section{INTRODUCTION}

Les poissons constituent une ressource alimentaire très importante en Afrique et dans le reste des pays en développement (HAYLOR et al., 1994 ; GAYE-SIESSEGGER, 2005). Selon ces auteurs, c'est souvent la seule source de protéines accessibles dans les zones rurales et urbaines. Toutefois, la satisfaction des besoins en poissons des populations humaines reste encore difficile à cause de la déficience des produits de pêche, consécutive à la surexploitation des stocks des ressources naturelles aquatiques (TACON, 2004 ; MARRA, 2005).

En Côte d'Ivoire, par exemple, la consommation moyenne de poissons est de 1 g/ind./j au Nord contre $24 \mathrm{~g} / \mathrm{ind} . / \mathrm{j}$ au Sud (Da COSTA et al., 1998). Face à la difficulté d'approvisionnement en poissons, la pisciculture est apparue comme une voie possible pour réduire le déficit de consommation de protéines animales (LAZARD et al., 1991; NAYLOR et al., 2000 ; FAO, 2004). Un peu partout en zone tropicale et sub-tropicale on assiste de ce fait à une intensification de l'élevage du tilapia du Nil Oreochromis niloticus avec une utilisation courante et importante des aliments artificiels pour son alimentation (FITZSIMMONS, 2000 ; HUECHT, 2000 ; EDWARDS et al., 2000 ; GAYE-SIESSEGGER, 2005). Toutefois, GREEN (1992), NGUENGA et al. (1997), WILLIAMS et al. (2000); SIDDHURAJU et BECKER (2003) et LITI et al. (2005), indiquent que la contrainte majeure à l'émergence de la pisciculture est l'alimentation à cause de son coût élevé. L'alimentation artificielle en aquaculture est donc le domaine où les plus grandes investigations ont été effectuées. Cependant, LUQUET (1990), DIANA et al. (1991) et DIANA et al. (1994) ont averti que les résultats déjà acquis sont à relativiser à cause des apports quantitatifs et qualitatifs des nourritures naturelles issues des structures d'élevage ouvertes. En effet, DIANA et al. (1994), TACON et De SILVA (1997), SHRESTHA et KNUD-HANSEN (1994), AZIM et al. (2001) ; AZIM et al. (2003) ont également mentionné que les nourritures naturelles rencontrées abondamment dans les milieux d'élevage constituent un apport non négligeable. Ces auteurs signalent que les organismes planctoniques se développement dans les étangs de l'aquaculture et sont facilement consommés par les poissons d'élevage. BEVERIDGE et BAIRD (2000), HUCHETTE et al. (2000) et KÖPRÜCÖ et ÖZDEMIR (2005) ont aussi signalé, qu'Oreochromis niloticus se nourrit aussi bien de la microflore et microfaune naturelles, que des aliments supplémentaires exogènes. Ces auteurs ont donc suggéré la prise en compte de ces interférences pour acquérir les bases nécessaires à une bonne gestion des matières premières et de la distribution des rations alimentaires journalières, ce qui devrait permettre une gestion appropriée de l'aliment exogène en vue de réduire les pertes et donc les coûts. Jusqu'à ce jour, les études de l'estimation de la quantité de nourritures naturelles et artificielles consommées par Oreochromis niloticus en captivité sont rares. Les 
travaux déjà effectués sont rares, et n'ont porté que sur les stades juvéniles (TOGUYENI et al., 1997 ; RICHTER et al., 2002 ; 2004). La présente étude semble être la première du genre, aussi précis sur les alevins et les adultes d'Oreochromis niloticus, élevés dans un milieu semi naturel. L'objectif visé est d'estimer les variations de la quantité de nourritures naturelles et artificielles consommées par Oreochromis niloticus en milieu semi naturel par les alevins, les juvéniles et les adultes.

\section{MATERIEL ET METHODES}

\section{Structures d'élevage et poissons expérimentaux}

Les essais, qui ont porté sur le tilapia du Nil Oreochromis niloticus, ont été réalisés à la ferme piscicole de Blondey, située à environ 25 kilomètres d'Abidjan (Côte d'Ivoire).dans quatre bassins bétonnés $\left(2 \times 1,5 \times 0,6 \mathrm{~m}\right.$, volume d'eau utile de 1,2 $\left.\mathrm{m}^{3}\right)$ et dans huit étangs en terre rectangulaires. Des étangs de $600 \mathrm{~m}^{2}$ pour le pré-grossissement et $800 \mathrm{~m}^{2}$ pour le grossissement ont été utilisés. Ces étangs avaient une profondeur de $0,7 \mathrm{~m}$ à l'arrivée d'eau dans les structures d'élevage et $1,25 \mathrm{~m}$, au moine. Toutes ces structures étaient munies d'un système de renouvellement continu d'eau et étaient alimentées par gravité à partir d'un barrage de retenue d'eau.

Les poissons échantillonnés étaient des alevins de $6 \mathrm{~g}$, des juvéniles d'environ $30 \mathrm{~g}$ et des adultes de $250 \mathrm{~g}$. Ces poissons étaient donc issus respectivement, des phases d'alevinage, de pré-grossissement et de grossissement. Les poissons ont été nourris avec un aliment composé de type pulvérulent contenant $25 \%$ de protéines.

\section{Conduite des élevages}

Les rations alimentaires quotidiennes ont été servies manuellement à la volée en deux repas ( $9 \mathrm{~h}$ et $15 \mathrm{~h}$ ). Des rations de $10 \%, 5 \%$ et $3 \%$ du poids total vif estimé ont été appliqués respectivement, en phases d'alevinage, de pré-grossissement et de grossissement conformément à la procédure de $\mathrm{Yl}$ et al. (2002). Des densités respectives de 500 ind. $/ \mathrm{m}^{2}, 13 \mathrm{ind} . / \mathrm{m}^{2}$ et $1,7 \mathrm{ind} . / \mathrm{m}^{2}$ ont été employées pour la production des alevins, des juvéniles et des adultes.

\section{Echantillonnage des poissons}

L'estimation de la ration journalière de nourriture repose sur la connaissance des variations journalières du poids du contenu de l'estomac de poissons d'une taille et d'âge connus (JARRE et al, 1991). Des captures ont été effectuées toutes les $3 \mathrm{~h}$, sur $24 \mathrm{~h}$ dans 4 bassins bétonnés (alevinage) et dans 8 étangs dont 4 étangs par phase de croissance. Deux cycles nycthéméraux de prélèvements d'échantillons des populations de poissons élevés ont été exécutés. Ainsi, toutes les trois heures, 20 poissons ont été capturés par structure et par phase d'élevage (alevinage, pré-grossissement et grossissement). Ceci fait un total à 160 poissons par cycle de $24 \mathrm{~h}$ et pour chaque stade de croissance. Le premier échantillonnage a eu lieu après la première distribution d'aliment de la journée ( 9 h). Les poissons prélevés ont été pesés et disséqués in situ. Les estomacs extraits ont été fixés au formol à $5 \%$, puis conservés individuellement.

\section{Analyse des contenus stomacaux}

Au laboratoire, les estomacs ont été ouverts sur toute leur longueur. Ensuite, leur contenu a été minutieusement extrait et pesé à l'aide de balance de marque SARTORIUS (précisions 0,001 g). Les différentes particules alimentaires (aliments distribués et naturels du milieu) ont été identifiées et séparées sous une loupe binoculaire de type OLYMPUS modèle SZ 30 et sous objectif microscopique de type Olympus BX 40 (grossissement $40 \mathrm{x}$ à 100x) selon les méthodes de RICHTER et al. (2004) et FOCKEN et al. (1998). Chacune des deux catégories de nourritures a été regroupée et pesée. 
Les poids frais de celles-ci ont été traités à l'aide du logiciel MAXIMS conformément à JARRE et al. (1990; 1991) et RICHTER et al. (2004). A partir des observations sur les variations journalières du poids des contenus stomacaux, ce logiciel calcule l'ingestion horaire de nourriture et, de là, la ration alimentaire journalière éventuellement divisée en aliments naturels et artificiels, comme dans le cas de la présente étude. La proportion relative de chacun des deux items présents dans l'estomac des poisons a été exprimée en pourcentage du total consommé.

\section{RESULTATS}

\section{Variations nycthémérales des proportions relatives des nourritures naturelles et artificielles du contenu stomacal des alevins, juvéniles et adultes d'Oreochromis niloticus en captivité}

Les aliments rencontrés dans l'estomac des poissons sont regroupés en deux fractions. La première est constituée des aliments distribués (apports exogènes) et la seconde rassemble les ressources naturelles du milieu d'élevage (apports endogènes). Les figures 1 ( $a$ et $b$ ) montrent les variations des proportions de ces deux catégories de nourritures dans le bol alimentaire sur $24 \mathrm{~h}$ selon les stades de développement. Aux heures de distribution des rations alimentaires journalières (9h et $15 \mathrm{~h}$ ) les nourritures artificielles sont plus importantes que les ressources naturelles. En dehors de ces périodes de nourrissage, les apports endogènes (nourritures naturelles) sont plus abondants, environ le triple ou le double de la quantité des aliments distribués. Cette tendance est plus marquée chez les alevins. Contrairement aux alevins, chez les juvéniles entre $18 \mathrm{~h}$ et $24 \mathrm{~h}$, les deux types de nourritures (artificielles et naturelles) sont présents dans les estomacs dans des proportions quasi identiques. En revanche, chez les adultes (phase de grossissement), les nourritures distribuées sont plus importantes entre $9 \mathrm{~h}$ et $24 \mathrm{~h}$. A l'opposé, au-delà de cette période (24h à $9 \mathrm{~h})$, le bol alimentaire est constitué dans son immense majorité de nourritures naturelles pour les trois stades de croissance.

\section{Rythme de la prise des nourritures chez les alevins, sub-adultes et adultes d'Oreochromis niloticus en captivité}

Les courbes représentant les variations nycthémérales de la prise de nourritures artificielles et naturelles obtenues à l'aide du logiciel MAXIMS sont présentées sur les figures 2 (a cycle 1 et b cycle 2). Ces courbes ont la même tendance pour les trois stades de croissance étudiés.

En phase d'alevinage, la prise de nourriture naturelle survient de $5 \mathrm{~h}$ à $12 \mathrm{~h}$ et de $15 \mathrm{~h}$ à $18 \mathrm{~h}$, avec deux sommets à $12 \mathrm{~h}$ et à $18 \mathrm{~h}$. Le poids des nourritures recueillies dans le contenu de l'estomac à ces deux moments ( $12 \mathrm{~h}$ et à $18 \mathrm{~h}$ ) est maximal : environ 0,07 à $0,08 \mathrm{~g}$. Au sujet des aliments apportés (nourritures artificielles), deux pics sont observés à $9 \mathrm{~h}$ et $15 \mathrm{~h}$, correspondant aux périodes de nourrissage. Le poids des aliments exogènes est alors maximal : 0,12 à $0,14 \mathrm{~g}$.

Chez les sub-adultes, la consommation des nourritures naturelles se déroule de $4 \mathrm{~h}$ à $6 \mathrm{~h}$ et de $10 \mathrm{~h}$ à $12 \mathrm{~h}$. Les poids maximum d'aliment naturel sont d'environ $0,25 \mathrm{à} 0,35 \mathrm{~g}$; ils sont observés à 6 heures et 12 heures. Les poids les plus élevés des aliments distribués $(0,4$ à $0,45 \mathrm{~g})$ ont été observés aux périodes de distribution des rations alimentaires journalières c'est-à-dire à $10 \mathrm{~h}$ et à $15 \mathrm{~h}$.

Chez les adultes, les poids de nourritures naturelles sont croissants de $4 \mathrm{~h}$ à $10 \mathrm{~h}$ et de $12 \mathrm{~h}$ à $16 \mathrm{~h}$. Les plus grandes quantités d'aliments naturels présents dans les estomacs ont été observées à $10 \mathrm{~h}(1,1 \mathrm{~g})$ et à $16 \mathrm{~h}(1,2 \mathrm{~g})$. Inversement, de $10 \mathrm{~h}$ à $12 \mathrm{~h}$ et de $16 \mathrm{~h}$ à $4 \mathrm{~h}$ les poids des aliments endogènes dans les estomacs sont décroissants. Les plus faibles valeurs $(0,1$ à $0,2 \mathrm{~g})$ ont été relevées à $4 \mathrm{~h}$. Pour les aliments distribués (nourritures artificielles), les poids les plus élevés (environ 2,4 à 3,3 g) ont été enregistrés à $9 \mathrm{~h}$ et à $15 \mathrm{~h}$, périodes de distribution des rations alimentaires journalières. Les plus faibles poids (environ 0,6 à $0,7 \mathrm{~g}$ ) de ces mêmes aliments ont été notés à $6 \mathrm{~h}$. 

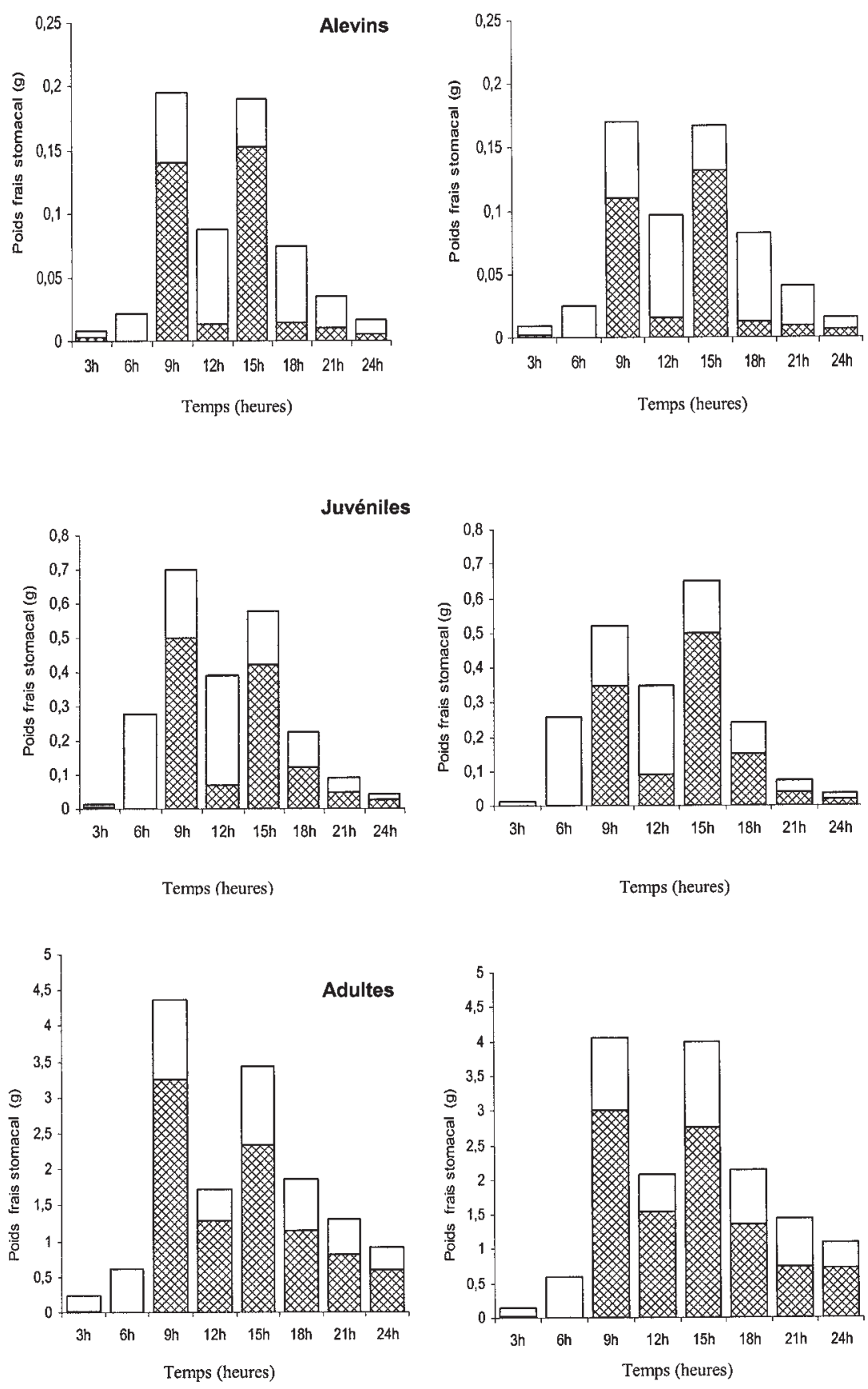

$\otimes$ nourriture artificielle

$\square$ nourriture naturelle

\section{Figure 1}

Abondances relatives nycthémérales des nourritures artificielles (exogènes) et naturelles (endogènes) dans le contenu de l'estomac chez les alevins les juvéniles et les adultes d'Oreochromis niloticus sur deux cycles de 24h. Cycle 1 à gauche ; cycle 2 à droite.

\section{Figure 1}

Relative abundance of the artificial food (exogenous) and natural (endogenous) in the gut contents of the juveniles, sub-adults and adults of Oreochromis niloticus over two cycles of 24 hours period. Cycle 1 on the left; cycle 2 on the right. 

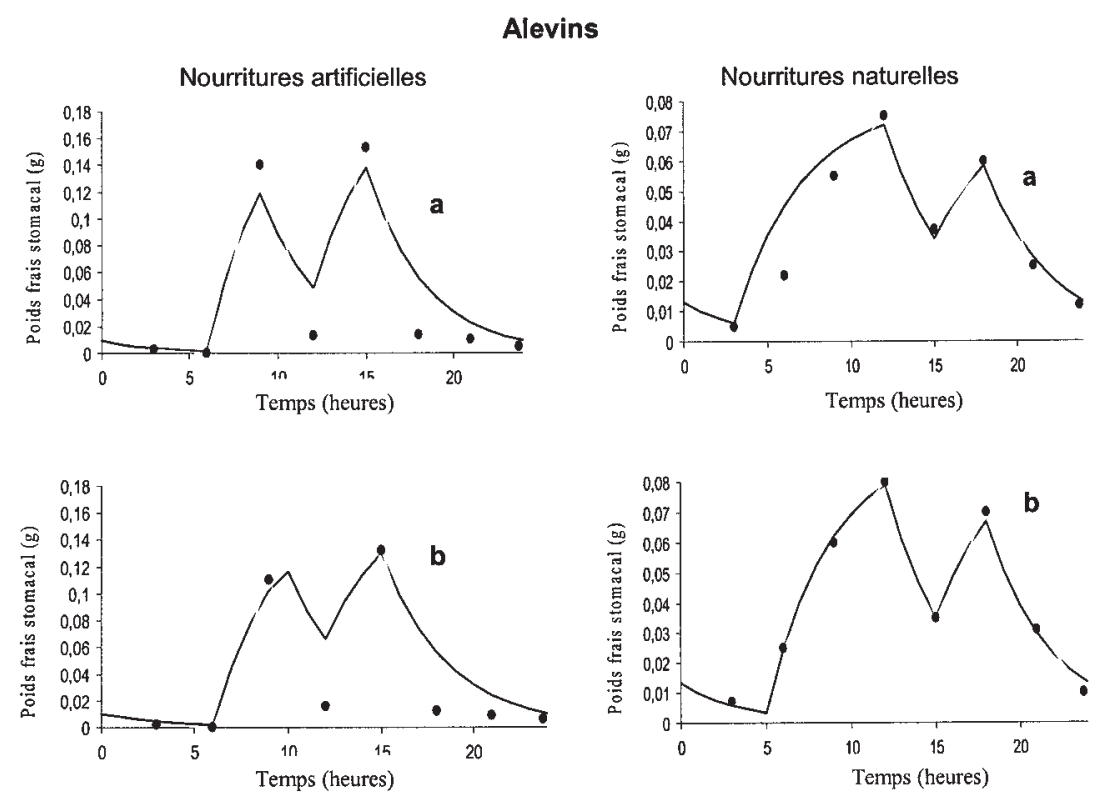

Juvéniles

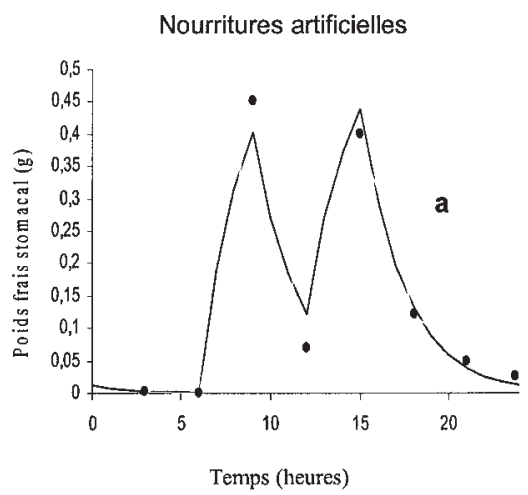

Nourritures naturelles
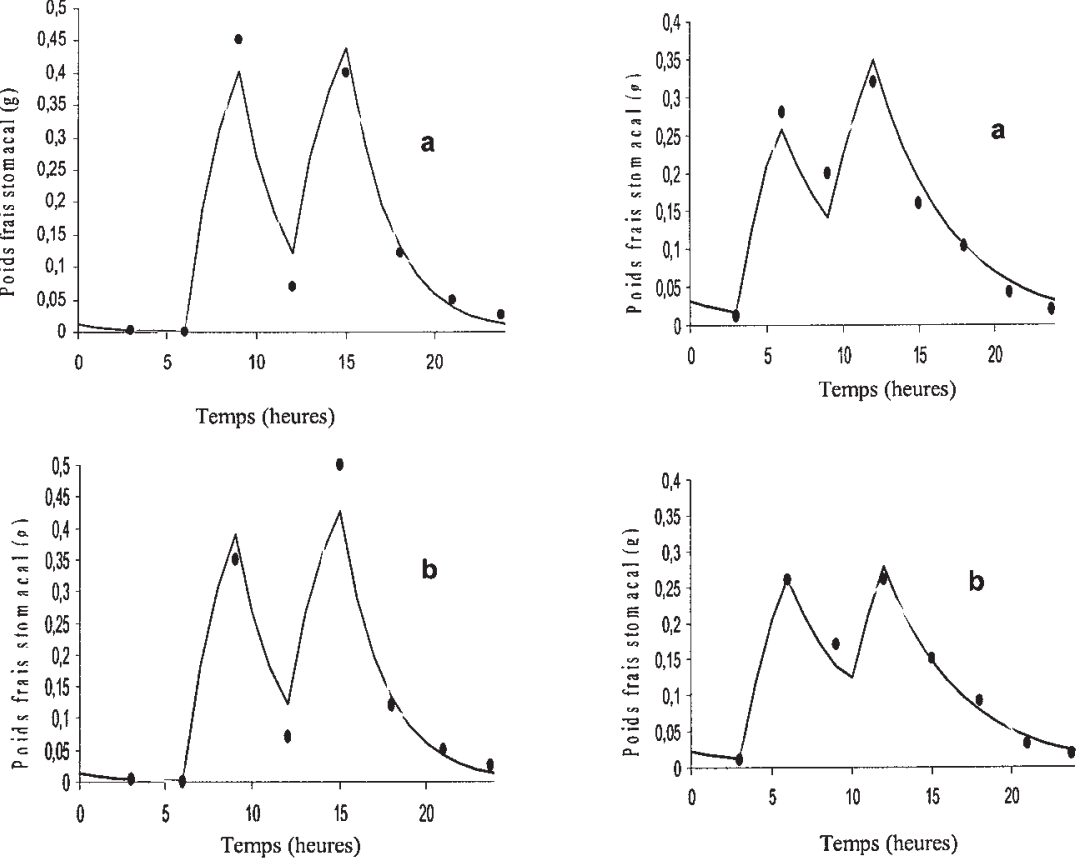

Figure 2

Courbes MAXIMS du rythme de la prise des nourritures artificielles et naturelles chez les alevins, les juvéniles et les adultes d'Oreochromis niloticus sur deux cycles de $24 \mathrm{~h}$, à la ferme de Blondey. (a) cycle 1 (figure au-dessus); (b) cycle 2 (figure au-dessous) ; ( $\bullet$ : valeurs observées ; $(\sim)$ : courbes ajustées.

Figure 2

MAXIMS curves of the consumption pattern of the artificial and natural food for the adults of Oreochromis niloticus on two cycles of $24 \mathrm{~h}$ at the Blondey farm. (a) cycle 1 (above) ; (b) cycle 2 (below) ; (•) : values observed ; ( ) : fitted curves. 


\section{Adultes}
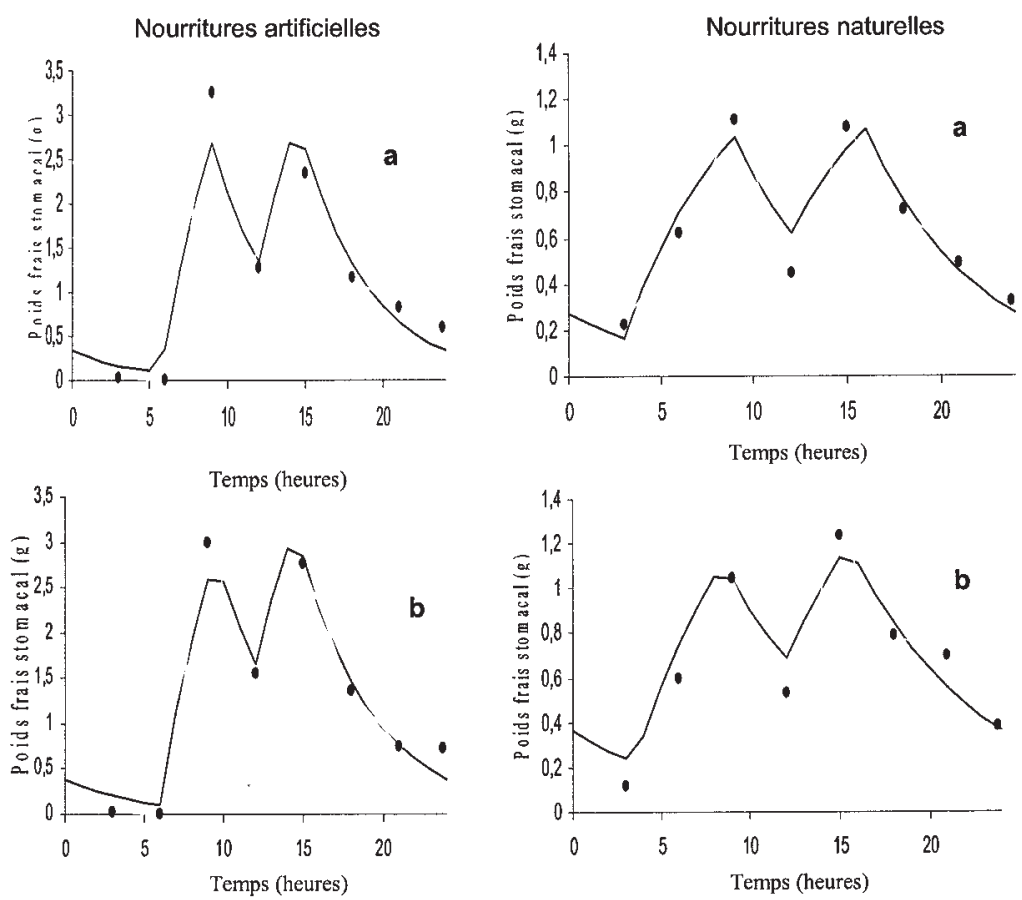

\section{Tableau I}

Organismes phytoplanctoniques (\%) rencontrés dans les contenus d'estomac d'Oreochromis niloticus aux stades d'alevinage, de pré-grossissement et de grossissement à la ferme expérimentale de Blondey.

\section{Table I}

Organisms of phytoplankton (\%) observed in the gut contents of the juveniles, sub-adults and adults of the Nile tilapia Oreochromis niloticus in the Blondey experimental fish farm.

\begin{tabular}{l|ccc|ccc}
\hline & \multicolumn{3}{|c|}{ Cycle 1 } & \multicolumn{3}{c}{ Cycle 2 } \\
\cline { 2 - 7 } & Alevin & juvénile & Adulte & Alevin & juvénile & Adulte \\
\hline Chlorophytes & 33 & 27,6 & 36,5 & 30 & 30 & 31 \\
Cyanobactéries & 17 & 42,4 & 47,5 & 21 & 38,57 & 57 \\
Euglénophytes & 29 & 21 & 12 & 29 & 20,82 & 10 \\
Chromophytes & 21 & 9 & 4 & 20 & 10,61 & 2 \\
\hline
\end{tabular}

Paramètres de consommation et importances relatives des ressources naturelles et artificielles dans la ration journalière des alevins, juvéniles et adultes d'Oreochromis niloticus

Les organismes du phytoplancton des contenus stomacaux sont présentés dans le tableau I. Les rations alimentaires journalières et les paramètres de consommation estimés sont consignés dans les tableaux II ( $a$ et b), tout comme les proportions des deux catégories de nourritures (endogènes et exogènes). 
Chez les alevins, les quantités des nourritures naturelles et artificielles consommées par jour sont respectivement d'environ $0,25 \mathrm{~g}$ et $0,36 \mathrm{~g}$, soit par heure, des quantités respectives de 0,03 g et 0,06 g pour les nourritures naturelles et artificielles. Les nourritures endogènes ingérées représentent environ $4 \%$ de leur poids vif contre $6 \%$ pour les nourritures artificielles. La ration alimentaire journalière totale estimée pour ces alevins est d'environ $10 \%$ de leur poids. La quantité totale d'aliment distribué a été de $10 \%$ de leur poids. La nourriture distribuée et non consommée par les poissons est donc de $40 \%$ du total distribué. La proportion des apports relatifs en aliments endogènes (tableaux II a et b) est de $40 \%$ (versus $60 \%$ pour la nourriture artificielle).

Au sujet des juvéniles, les quantités consommées estimées des ressources naturelles du milieu d'élevage et exogènes varient respectivement de 0,6 à $0,7 \mathrm{~g}$ et de 1,3 à 1,32 $\mathrm{g}$ par jour. Les quantités estimées respectives d'aliments ingérés et par poisson sont de $0,22 \mathrm{~g} \cdot \mathrm{h}^{-1}$ pour les nourritures distribuées et de $0,12 \mathrm{~g} \cdot \mathrm{h}^{-1}$ pour nourritures naturelles (au total $\left.0,34 \mathrm{~g} \cdot \mathrm{h}^{-1}\right)$. Les nourritures artificielles ingérées représentent environ 4,36 à 4,45\% de leur poids vif (versus 2,05 à 2,31\% pour les nourritures naturelles). La ration alimentaire journalière totale estimée pour ces poissons est approximativement de 6,5 à 6,67\% de leur poids. Au cours des expériences, les poissons ont été nourris à $5 \%$ de leur poids. La quantité de nourriture distribuée et non consommée par les poissons est d'environ $12 \%$ du total distribué. La comparaison des proportions des apports relatifs en aliments endogènes et exogènes montre que les nourritures artificielles sont plus importantes (environ 65 à $68 \%$ du total consommé) que les nourritures naturelles (approximativement 32 à $35 \%)$.

Chez les adultes, la quantité de ressources naturelles consommée par jour varie de $2,47 \mathrm{~g}$ à $2,6 \mathrm{~g}$ contre 6,61 à 6,9 g pour les aliments artificiels. La quantité totale d'aliments ingérés est estimée à $9,3 \mathrm{~g}$. Soit, une consommation horaire d'environ $1,15 \mathrm{~g}$ pour les apport exogènes et de 0,26 à $0,28 \mathrm{~g}$ pour les apports endogènes (au total 1,41 g). Les nourritures artificielles ingérées représentent environ 2,63 à 2,69 \% du poids vif des poissons (versus 0,97 à 1,03 \% pour les ressources naturelles). La ration alimentaire totale par jour de ces poissons est donc approximativement de 3,61 à 3,69 \% de leur poids. Les poissons ont été nourris à raison de $3 \%$ de leur poids au cours de l'expérimentation. L'aliment distribué et non ingéré par les poissons est par conséquent d'environ $12 \%$ du total distribué. De l'analyse de ces résultats, on constate que les aliments artificiels représentent 72 à $74 \%$ de la ration ingérée contre 26 à $28 \%$ pour les aliments naturels.

\section{DISCUSSION}

Le rythme de la prise de nourritures naturelles et les variations nycthémérales quantitatives du poids de l'estomac révèlent des activités alimentaires diurnes et périodiques. Les résultats de notre étude sont en accord avec ceux rapportés par RICHTER et al. (2004) obtenus aux Philippines. En effet, ces auteurs indiquent qu'Oreochromis niloticus a une activité alimentaire essentiellement diurne. Toutefois, ils signalent que ce poisson peut présenter des prises nocturnes d'aliments.

De façon prévisible, les apports en aliments exogènes sont plus abondants que les nourritures naturelles dans les contenus stomacaux aux heures de la distribution des rations journalières ( $9 \mathrm{~h}$ et $15 \mathrm{~h}$ ). Des contributions respectives de nourritures naturelles d'environ 26 à $28 \%, 32$ à $35 \%$ et 40 à $42 \%$ ont été enregistrées lors du grossissement, du pré-grossissement et de l'alevinage. Pour les aliments artificiels distribués dans le même ordre, les proportions observées sont comprises entre 72 et $74 \%, 65$ et $68 \%$, puis 58 et $60 \%$. Des proportions de ressources naturelles plus élevées (50\%) obtenues en étang ont été rapportées par HASTINGS et DICKIE (1972). Cependant, ces auteurs n'ont pas mentionné le stade de croissance étudié. Ces différents résultats semblent indiquer la complémentarité entre les ressources naturelles issues du milieu d'élevage 


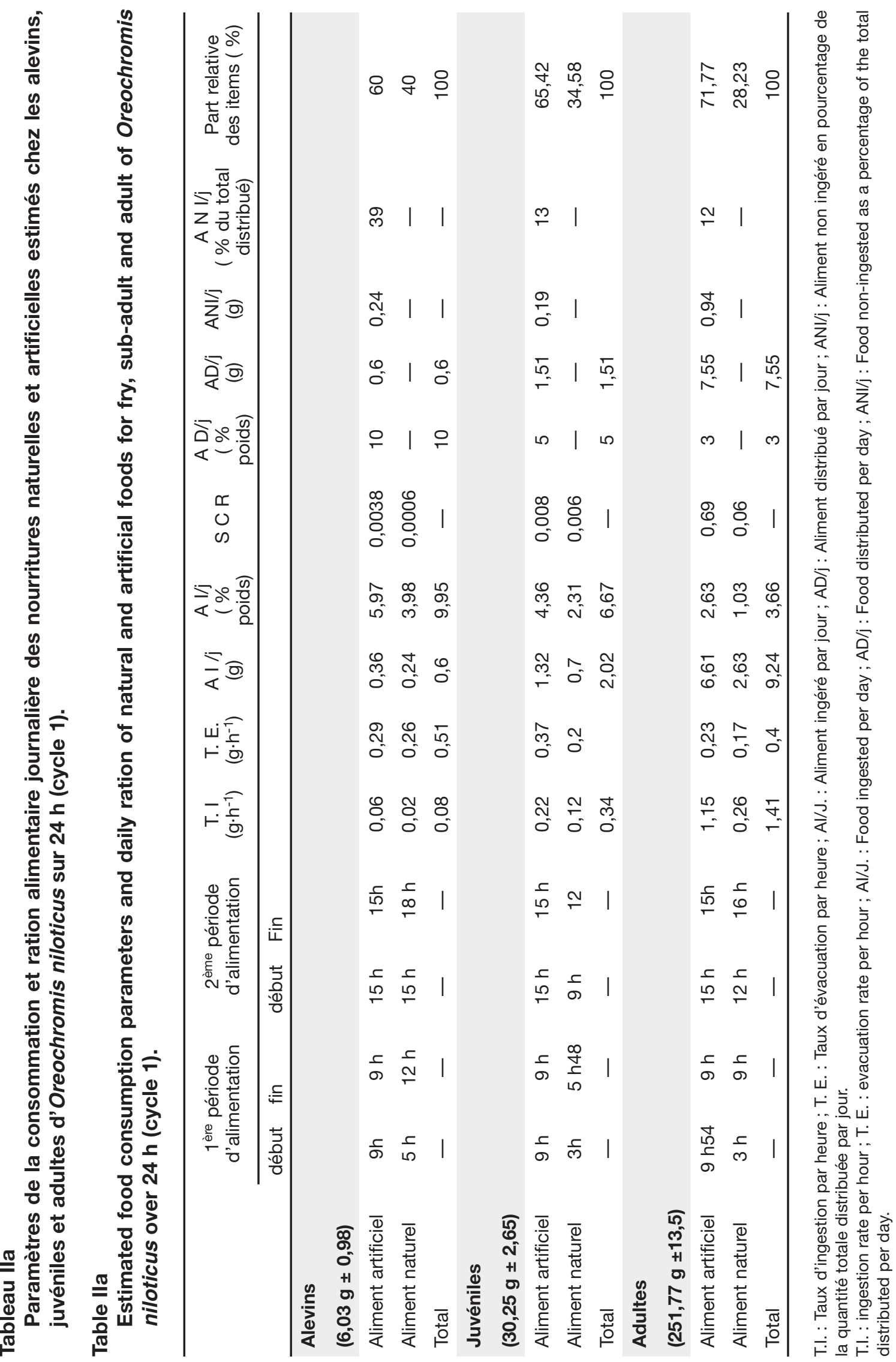




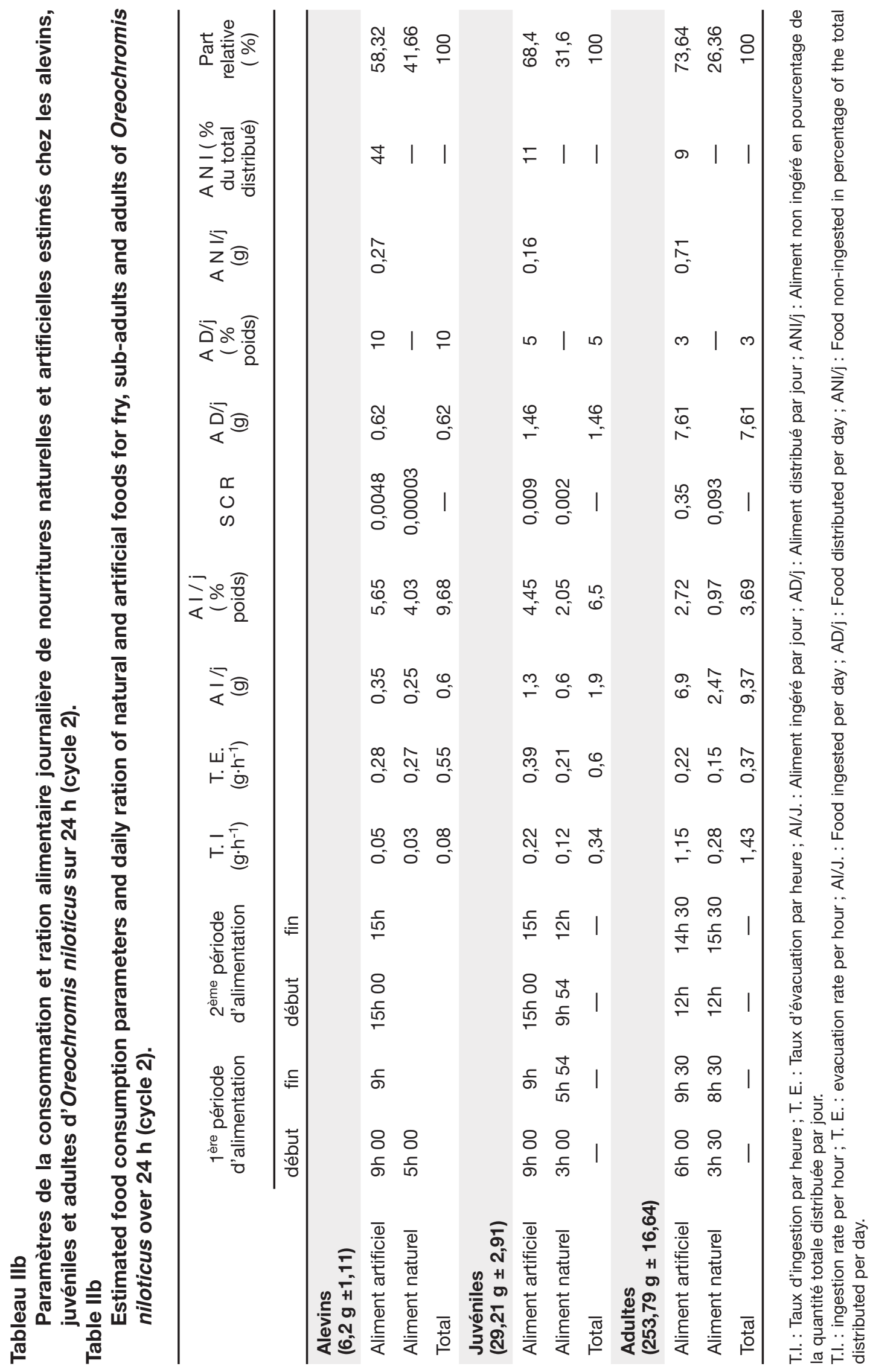


et les aliments artificiels apportés dans l'alimentation de ce poisson. Selon DIANA et al. (1994), WAIDBACHER et al. (2006), les nourritures naturelles issues du milieu d'élevage contribuent à une augmentation de la croissance chez Oreochromis niloticus à des ratios compris entre $45 \%$ et $74 \%$. Comme l'a indiqué DE SILVA (1993), ces ressources naturelles riches en protéines (55 à $60 \%$ ) et peu énergétiques contribueraient à couvrir les besoins azotés des poissons en élevage semi-intensif. Par conséquent, l'utilisation d'aliments artificiels à bases de sous-produits agricoles ( $25 \%$ de protéines brutes) en association avec des apports de 30 à $40 \%$ en ressources endogènes permet de rehausser la teneur protéique des aliments consommés vers des valeurs comprises entre 35 et $45 \%$. Nos résultats montrent que les proportions des nourritures naturelles prélevées dans le milieu décroissent de l'alevinage au grossissement. Cette différence de régime alimentaire observée entre les stades de développement pourrait être en relation avec les besoins nutritionnels requis pour chaque stade de croissance. LEE et PUTMAN (1973), MORIARTY et MORIARTY (1973), SHIAU et PENG (1993), BOWEN (1982), BOWEN et al. (1995), STICKNEY (1996) et FITZSIMMONS (1997) abondent dans le même sens. Ces auteurs ont rapporté que les adultes de cette espèce ou d'autres poissons ont besoin d'aliments très énergétiques et peu protéiques pour leur métabolisme et leur croissance. De leur côté, PERSON-LE RUYET et BERGOT (1999) mentionnent que les larves et les juvéniles requièrent plutôt des aliments peu glucidiques, mais riches en protéines, en vitamines (plancton), en lipides et en minéraux pour une croissance rapide. Les proportions d'aliments artificiels distribués et non consommés sont respectivement de $40 \%$ du total distribué chez les alevins et de $12 \%$ chez les juvéniles et les adultes. ACOSTA-NASSAR et al. (1994) et GROSS et al. (2000) ont indiqué que 5 à $30 \%$ des aliments distribués sont souvent perdus et ne profitent pas directement aux poissons d'élevage. Cette importante perte d'aliment pourrait s'expliquer par l'effet de densité de mise en charge et de la compétition alimentaire. En effet, pour avoir accès à l'aliment distribué, le déplacement des poissons occasionne des mouvements qui pourraient entraîner une importante perte, car les aliments étaient de type pulvérulent. EWING et al. (1998) confirment cette hypothèse. Ces auteurs ont rapporté que la compétition alimentaire croît avec la densité de mise en charge et influe considérablement sur la disponibilité de l'aliment à consommer par les poissons.

Par ailleurs, les résultats montrent une variation qualitative des éléments phytoplanctoniques dans le régime alimentaire des alevins, des juvéniles et des adultes (prédominance des Chlorophytes et cyanobactéries chez les premiers, des Euglenophytes et Chlorophytes, chez les juvéniles et les adultes). Ceci a-t-il une influence sur la place de la nourriture naturelle dans l'alimentation des poissons ? Ce point mériterait une étude complète.

\section{CONCLUSION}

Cette étude sur la production du tilapia du Nil Oreochromis niloticus dans les structures ouvertes (pisciculture semi-intensive) souligne les apports relatifs aux nourritures naturelles et artificielles. Les proportions des nourritures naturelles consommées ont été d'environ $4 \%, 2,31 \%$ et $1 \%$ contre $6,4,45$ et 2,7 \% pour l'aliment artificiel respectivement chez les alevins, les sub-adultes et les adultes. Dans ce même ordre de stade de croissance indiquée, les rations alimentaires quotidiennes estimées sont approximativement de $10 \% ; 6,7 \%$ et $3,7 \%$ du poids vif.

\section{REMERCIEMENTS}

Ce travail a été réalisé dans le cadre du Programme d'Appui à la Recherche Agronomique (PARA), financé par la Mission de Coopération française et l'ONG NGH. Nous leur adressons donc nos sincères remerciements. Nous tenons aussi à remercier 
le Ministère de l'Enseignement Supérieur et le Dr S. ANGELICA, responsable de l'ONG $\mathrm{NGH}$, pour leur soutien financier. Nous voudrions ici, exprimer notre gratitude à l'Unité de la Recherche et de l'Enseignement Supérieur de Korhogo (URES-korhogo) de l'Université de Bouaké pour son assistance technique qui a permis le déroulement des travaux de recherche. Enfin, nous tenons à remercier M. K.A. DETOH propriétaire de la ferme Blondey qui a servi de base pour nos expérimentations. Nous remercions également le $\mathrm{Dr} \mathrm{K}$. Sebastino Da COSTA, chargé de recherche dans le Programme Pêche et Aquaculture du Centre National de Recherche Agronomique (CNRA), pour la lecture critique de ce travail. Enfin, les auteurs remercient les lecteurs du journal qui ont aidé à la mise en forme finale de ce manuscrit.

\section{BIBLIOGRAPHIE}

ACOSTA-NASSAR M. V., MORELL J. M., CORREDOR J. E., 1994. The nitrogen budget of a tropical semi-intensive freshwater fish culture pond. Journal of World Aquaculture Society. 25, 261-270.

AZIM M.E., WAHAB M. A., VANDAM A.A., BEVERIDGE M.C.M., MILSTEIN A., VERDEGEM M.C.J., 2001. Optimization of fertilization rates for maximizing epiphyte growth on artificial substrates and implications for epiphyte-based aquaculture. Aquaculture Research, 32, 749- 760.

AZIM M.E., VERDEGEM M.C.J., MANTINGH I., VAN DAM A.A., BEVERIDGE M.C.M., 2003. Ingestion and utilization of periphyton grown on artificial substrates by Nile tilapia Oreochromis niloticus L. Aquaculture Research, 34, 85- 92.

BEVERIDGE M.C.M., BAIRD D. J. 2000. Diet feeding and digestive physiology. In : BEVERIDGE M. C. M. and MCANDREW B. J. (Eds.). Tilapias: Biology and Exploitation. Kluwer Academic Publishing (Dordrecht), 9-81.

BOWEN S. H., 1982. Feeding, digestion and growth-qualitative considerations. In : PULLIN R. S. V. and LOWE-McCONNELL R. H. (Eds.). The Biology and Culture of Tilapias. ICLARM, Conferences Proceedings, Manilla, 141- 156.

BOWEN S. H., LUTZ E.V., AHLGREN M. O., 1995. Dietary protein and energy as determinants of food quality : trophic strategies compared. Ecology, 76, 899-907.

DA COSTA K.S., TRAORE K., TITO DE MORAIS L., 1998. Effort de pêche et production exploitée dans les petites retenues du nord de la Côte d'lvoire. Bull. Fr. Pêche Piscic., 348, 65-78.

DE SILVA S. S., 1993. Supplementary feeding in semi-intensive aquaculture systems. In : NEW M. B., TACON A. G. J. et CSAVAS I (Eds.). Farm Made Aquafeeds. Proceedings of the FAO/AADCP, Bangkok, Thailand. FAO, Rome, 14- 60.

DIANA J. S., LIN C. K., SCHNEEBERGER P. J., 1991. Relationships among nutrient inputs, water nutrient concentrations, primary production, and yield of Oreochromis niloticus in ponds. Aquaculture, 92, 323-341.

DIANA J. S., LIN C. K., JAIYEN K., 1994. Supplemental feeding of tilapia in fertilized ponds. Journal of the World Aquaculture Society, 25, 497-506.

EDWARDS P., LIN C. K. YAKUPITIYAGE A., 2000. Semi-intensive pond aquaculture. In : BEVERIDGE, M. C. M., MCANDREW, B. J. (Eds.), Tilapias : Biology and Exploitation. Kluwer Academic Publishing, 377-403.

EWING R. D., SHEAHAN J. E., LEWIS M. A. PALMISANO A. N., 1998. Effects of rearing density and raceway conformation on growth, food conversion, and survival of juvenile spring Chinook salmon. Program Fish-Culture., $60 \mathrm{p}$.

FAO, 2004. The State of World fisheries and Aquaculture (SOFIA). FAO Corporate Document Repository. www.fao.org. 
FITZSIMMONS K., 1997. Introduction to tilapia nutrition. In: FITZSIMMONS K. (Ed.). Tilapia aquaculture. The International Symposium on Tilapia in aquaculture. ICLARM Conferences Proceedings, Florida, $9-12$.

FITZSIMMONS K., 2000. Tilapia: the most important aquaculture species of the $21^{\text {st }}$ century. In : FITZSIMMONS K. and FILHO J. C. (Eds.). Proceedings from the Fifth International Symposium on Tilapia in Aquaculture, Rio de Janeiro, 3 - 8.

FOCKEN U., GROTH A., COLOSO R. M., BECKER K., 1998. Contribution of natural food and supplemental feed to the gut content of Penaeus monodon Fabricus in a semiintensive pond system in the Phillippines. Aquaculture 164, 105 - 116.

GAYE-SIESSEGGER J., FOCKENA U., ABEL, BECKER H. J. K., 2005. Improving estimates of trophic shift in Nile Tilapia, Oreochromis niloticus (L.), using measurements of lipogenic enzyme activities in the liver. Comparative Biochemistry and Physiology, $140,117-124$.

GREEN B. W., 1992. Substitution of organic manure for pelleted feed in tilapia production. Aquaculture, 101, 213-222.

GROSS A., BOYD C. E., WOOD C. W., 2000. Nitrogen transformations and balance in channel catfish ponds. Aquaculture Engineering, 24, 1-14.

HASTINGS W. H., DICKIE L. M., 1972. Fish nutrition : feed formulation and evaluation. Academic. Press (London), 327- 374.

HAYLOR G., YOUNG J. A., MUIR J. F., SCOTT D.C.B., 1994. Commercial Aquaculture in Africa. CDC, London, $105 \mathrm{p}$.

HUCHETTE, S. M. H., BEVERIDGE, M. C. M., BAIRD, D. J., IRELAND, M., 2000. The impacts of grazing by tilapias (Oreochromis niloticus L.) on periphyton communities growing on artificial substrate in cages. Aquaculture, 186, 45- 60.

HUECHT T., 2000. Considerations on African aquaculture. World Aquaculture, 31, 12 - 19.

JARRE A., PALOMARES M. L., SORIANO M. L., SAMBILAY V. C., PAULY D., 1990. A user's manual for MAXIMS. A computer program for estimating the food consumption of fishes from diel stomach contents data and population parameters. JARRE A., PALOMARES M. L., SORIANO M. L., SAMBILAY V. C, PAULY D., 1991. "Some new analytical and comparative methods for estimating the food consumption of fishes." ICES Marine Science Symposium, 193, 99-108.

ICLARM software 4, International Center for Living Aquatic Resources Management, Manila, Philippine, $27 \mathrm{p}$.

KÖPRÜCÜ K., ÖZDEMIR Y., 2005. Apparent digestibility of selected feed ingredients for Nile tilapia (Oreochromis niloticus). Aquaculture, 250, 308 - 316.

LAZARD J., LECOMTE Y., STOMAL B., WEIGEL J. Y., 1991. Pisciculture en Afrique Sub-Saharienne. Situation et projets dans les pays francophones: Propositions d'actions. MCD, Paris, $156 \mathrm{p}$.

LEE D. J., PUTMAN G. B., 1973. The response of rainbow trout to varying protein : energy ratio in a test diet. Journal of nutrition, 103, $916-922$.

LITI D., CHEROP L., MUNGUTI J., CHHORN L., 2005. Growth and economic performance of Nile tilapia (Oreochromis niloticus L.) fed on two formulated diets and two locally available feeds in fertilized ponds. Aquaculture Research, 36, 746- 752.

LUQUET P., 1990. Alimentation des tilapias. In : LAZARD J., JALABERT et B. DOUDET T. (Eds.). L'aquaculture des tilapias. Du développement à la recherche. Bois et Forêts des Tropiques, 29 -38.

MARRA J., 2005. When will we tame the oceans ? Nature, 436, 175-176. 
MORIARTY C. M., MORIARTY D. J. W., 1973. Quantitative estimation of the daily ingestion of phytoplankton by Tilapia nilotica and Haplochromis nigripinnis in Lake George, Uganda. Journal of. Zoology (London), 17, 209 - 255.

NAYLOR R. L., GOLDBURG R. J., PRIMAVERA J. H., KAUTSKY N., BEVERIDGE M. C. M., CLAY J., FOLKE C., LUBCHENCO J., MOONEY H., TROELL M., 2000. Effect of aquaculture on world fish supplies (review article). Nature, 405, 1017- 1024.

NGUENGA D., BREINE J. J., SULEM YONG S., TEUGELS G. G., OLLEVIER F., 1997. Effect of animal manure and chemical fertilizer on the growth and survival of Tilapia cameronensis. Holly in Cameroon. Aquaculture Research, 28, 231-234.

PERSON-LE RUYET J., BERGOT P., 1999. Aliments inertes pour les larves de poisson. In : GUILLAUME J., KAUSHIK S., BERGOT P., METAILLER R. (Eds.), Nutrition et alimentation des poissons et crustacés, 285 - 296, INRA, Paris.

RICHTER H., FRANCIS G., BECKER K., 2002. A reassessment of the maintenance ration of red tilapia. Aquaculture International, 10, 1 - 9.

RICHTER H., GONZAL A., FOCKEN U. et BECKER K., 2004. Uptake of natural food and supplemental feed by cultured Nile tilapia, Oreochromis niloticus L., in Laguna de Bay, Philippines. In : BOLIVAR R., MAIR G. and FITZSIMMONS K. (Eds.), Proceedings, the $6^{\text {th }}$ International Symposium on Tilapia in Aquaculture, 347-362, ISTA, Manila.

SHIAU S. Y., PENG C. Y., 1993. Protein sparing effect by carbohydrates in diets for tilapia, Oreochromis niloticus x O. aureus. Aquaculture, 117, 327-334.

SHRESTHA M. K., KNUD-HANSEN C. F., 1994. Increasing attached microorganism biomass as a management strategy for Nile tilapia (Oreochromis niloticus) production. Aquaculture. Eng. 13, 101- 108.

SIDDHURAJU P., BECKER K., 2003. Comparative nutritional evolution of differentially processed mucuna seeds (Mucuna pruriens (L.) DC. Var. utilis (Wall ex Wight) (Baker ex Burck) on growth performance, feed utilization and body composition in Nile tilapia (Oreochromis niloticus L.). Aquaculture Research, 34, 487 - 500.

STICKNEY R. R., 1996. Tilapia update, 1995. World Aquaculture, 27 (1), 45 - 50.

TACON, A.G.J., 2004. Use of fish meal and fish oil in aquaculture : a global perspective. Aquatic Resources, Culture and Development, 1, 3-14.

TACON A. G. J., DE SILVA S. S., 1997. Feed preparation and feed management strategies within semi-intensive fish farming system in the tropics. Aquaculture, 151, 379404.

TOGUYENI A., FAUCONNEAU B., BOUJAR T., FOSTIER A., KUHN E. R. MOL K. A., BAROILLER J. F., 1997. Feeding behaviour and food utilization. -In tilapia, Oreochromis niloticus L. : Effects of sex ration and relationships with the endocrine status. Physiology. Behaviour. 62 (2), 273-279.

WAIDBACHER H., LITI D. M., FUNGOMELI M., KMBALUKA R., MUNGUTI J. M., STRAIF M., 2006. Influence of pond fertilization and feeding rate on growth performance, economic returns and water quality in a small-scale cage-cum-pond integrated system for production of Nile tilapia (Oreochromis niloticus L.). Aquaculture Research, 2006, 37, 594-600.

WILLIAMS M. J. J., BELL D., GUPTA M. V., DEY M., AHMED M., PREIN M., CHILD S., GARDINER P. R., BRUMMETT R., JAMU D., 2000. Responsible aquaculture can aid food problems. Nature, 406, 673.

YI Y., LIN C. K., DIANA J. S., 2002. Hybrid catfish (Clarias macrocephalus x C. gariepinus) and Nile tilapia (Oreochromis niloticus) culture in an integrated pen-cum-pond system : growth performance and nutrient budgets. Aquaculture, $217: 395-408$. 